\title{
Sensor Web Design Studies for Realtime Dynamic Congestion Pricing
}

\author{
Milton Halem*, Anand Patwardhan*, Sandor Dornbush*, Michael Seablom ${ }^{\dagger}$ and Yelena Yesha* \\ * Department of Computer Science and Electrical Engineering, UMBC, Baltimore, MD 21250 \\ Email: $\{$ halem, anand2, sandor1, yeyesha\}@cs.umbc.edu \\ $\dagger$ NASA Goddard Space Flight Centre, Greenbelt, MD 20771
}

Email: Michael.S.Seablomenasa.gov

\begin{abstract}
Traffic Congestion is a multi-billion dollar national problem and worsening every year with population growth and increase in freight traffic. We present a model for realistic simulation studies to mitigate congestion in urban areas using dynamic congestion pricing on express toll lanes. Specifically, we identify and address the design issues needed to develop a real time event driven sensor web observing system for traffic monitoring that provides dynamic congestion pricing. To assess the feasibility of this sensor web system, we are in the process of conducting simulation studies based on real data for various system configurations to validate the mitigating impact of dynamic congestion pricing on intermodal freight flow to and from the ports in urban areas. In this paper, we focus on freight flow into the Baltimore corridor from its ports.
\end{abstract}

\section{INTRODUCTION}

Congestion stands well above all the other factors as the principal public concern regarding transportation [4]. In particular, the continued growth in global trade is leading to increasing demand on ports to enable the rapid delivery and distribution of freight. The incoming freight handling capacity of ports is limited by existing road and rail infrastructures and their capacities. The urban traffic situation for the state of Maryland is forecast to become worse yearly as population and truck traffi c continue to increase in the Baltimore, MD corridor including Washington, D.C. Most State transportation authorities today face relatively constant budgets and are exploring innovative approaches to raise capital to stay abreast of inflation for major transportation projects addressing congestion. Express toll lanes and variable pricing is a current popular approach being considered by several states including MD. However, fi xed pricing as function of time of day (i.e. rush hour congestion) is the initial method MDOT has chosen for pricing of express toll lanes on several interstate highways under construction. MDOT would like to consider dynamic pricing [4] since it presumably can account for other causes of congestion such as accidents, weather, construction, maintenance, etc.; other than rush hour traffi $\mathrm{c}$ if it were cost effective and risk averse. Realistic simulation studies for the domain of freight fbw from ports is the first step in providing evidence to transportation agencies and the public that dynamic pricing is indeed a feasible congestion mitigating factor that can be cost effective with minimal risk.

In this paper, we present a sensor web design for dynamic congestion pricing and identify the observational input data streams required for model simulations. We then describe the generation of simulated traffic data for congested sites in the fbw of freight from the port of Baltimore based on acquired historical data from various archives. We will also consider congestion based pricing scenarios for potential future scenarios caused by natural or human induced events related to hurricanes, fi res, fbods, ice, fog, sporting events, parades, as well as road and bridge closings for construction maintenance.

\section{BACKGROUND}

Fixed pricing methods are being successfully deployed for freight fbw from the Los Angeles/Long Beach area as well as the Seattle ports. However, the changing technological landscape in the domains of wireless communication, sensor technology, and satellite sensor webs offers additional opportunities that can radically extend the static transportation pricing model from the rush hour scenario to help address the wider range of factors leading to 
congestion problems. We propose the integration of commercial satellite remote sensing, low cost UAVs, tracking devices installed on feets of trucks (e.g. VeriWise), automated remote GPS collections from individual vehicles, in addition to geo-based anchored sensors and traffi c monitoring devices like high defi nition cameras, to form a real-time sensor web to infer traffi $\mathrm{c}$ fbw and enable dynamic pricing schemes to mitigate congestion. The following section illustrates how this pre-requisite technology might be deployed as a dynamic pricing design model. These scenarios differ fundamentally from Los Angeles and Seattle tests currently being conducted employing static pricing systems applied at specifi c times and locations to address fi xed periods of congestion. In contrast, our proposed sensor web system proposed, monitors variable occurrences in time and location of congestion for near real time decision analysis for freight pricing based on a variety of unpredicted events. We explain by a scenario in the following section what measurements/sensor input are required in real time to modify express toll pricing to change the freight fbw in the Baltimore corridor based on model data assimilation and prediction schemes.

\section{An Intermodal Freight Flow Scenario at the Port of Baltimore}

Freight volumes have grown signifi cantly from the 90s and after a dip since 2001, have now regained pre-2001 levels and are expected to grow at more than $8 \%$ annually over the next several decades. Limited landside and waterside access limit the capacity of ports, and congestion at intermodal links to surface transportation like rail and roads exacerbate the problem. Increasing traffi c congestion at the ports and on the access routes prevent port facilities from operating at peak effi ciencies. Congestion points not only hinder operation effi ciency, but also inhibit growth of port operations. To enhance the safety, reliability, productivity and responsiveness of the freight transportation system it is necessary to address congestion in order to make use of existing capacity.

We have chosen as a typical urban location to illustrate how the proposed sensor web system might work upon completion for the Baltimore Port. We assume that the State of Maryland has instituted their planned 4 EZ Pass toll lanes, two in each direction alongside a 5 mile stretch of I-95 free lanes as well as one lane HOV toll lanes for the I-695 beltway. This system is planned as a static approach to congestion pricing based on time of day charges to mitigate traffi c congestion. We describe a scenario in Figure 1 in which we leverage this system for dynamic congestion pricing. Assume there is an accident during evening rush hour traffi c just South of the Fort McHenry tunnel on I-95. In the past 48 hours two large container ships have delivered two thousand containers to Seagirt docks. We also assume that the CSX rail at the Seagirt docks have instituted dynamic market pricing for their rail use at Seagirt. The shipping companies are keen to deliver those packages as soon as possible however if they are delivered immediately it will complicate the already bad congestion on I-95.

The congestion is detected by the reduced fbw at the Ft. McHenry toll plaza, road based sensors and reports from both VeriWise [3] and StreetSmart Traffi c [2] enabled navigational devices. All of this information is fed back to MDOT command and control centers. Based on this information, the command center raises the toll on I-95 to discourage the use of that route. At the same time the cost of other routes such as the I-895 Harbor Tunnel an I-695 Harbor Bridge reduced for freight to encourage alternate route choices by the freight truckers. In addition, CSX reduces their rail prices to offer incentives for additional use of their services. The congestion on I-95 and the associated fee change is advertised on electronic road signs, radio broadcasts and in it is transmitted to in vehicle navigational devices. The command center also launches a mini Unmanned Aerial Vehicle to monitor both the existing traffi $\mathrm{c}$ on I-95 and the alternate routes. This mini UAV will tell the command center when the traffi $\mathrm{c}$ has cleared on I-95 or when the alternate routes have become overloaded. Historical satellite data bases are queried to determine which tolls should be "incentivized" to detour traffi c to routes that previously were optimal for handling the overfbws freight fbws.

A truck driver with an in vehicle navigational device would get a message on their screen advising them that a cheaper route is available. The driver would have the choice to ignore the information, however most drivers will heed the advice and take the alternate routes of I-895 and I-695. This shifts a portion of the vehicle traffi c to routes that do not have current traffi c congestion. Through a variety of technologies it is possible to use each drivers self interest to use the road network most optimally.

Such systems need to integrate satellite remote sensing for broader area monitoring capabilities offered by a space based sensor webs together with spatial ground information technologies. Clearly, extensive and realistic simulations studies need to be conducted and their results validated prior to government agencies seriously adopting such systems. 


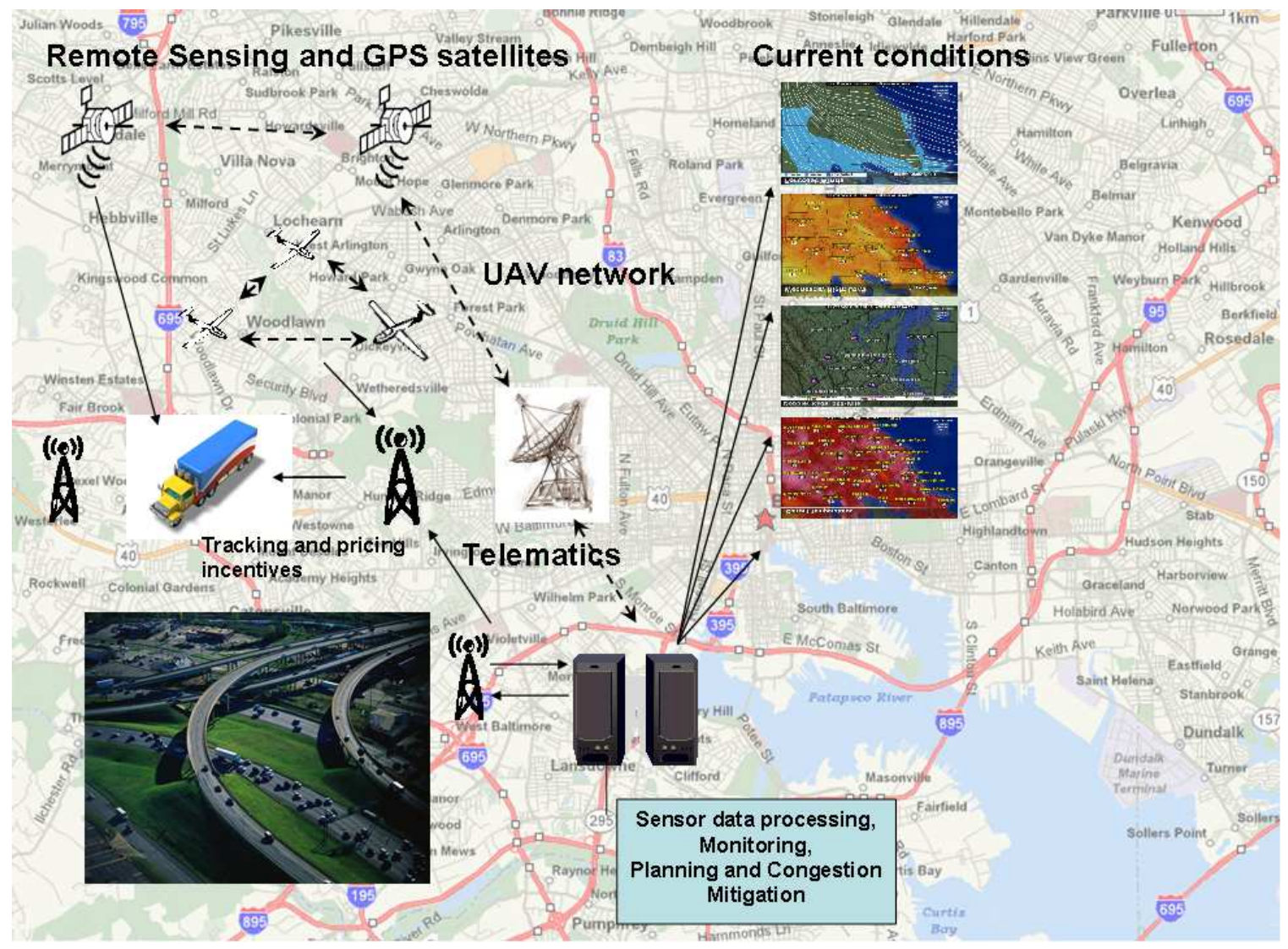

Fig. 1. A Typical Scenario

\section{Sensor Web Simulation for Dynamic Congestion Pricing}

A sensor web simulator (SWS) is used to conduct extensive design trade-off studies with the DynaMIT traffi c model employing real time input from actual traffi c patterns. Input case data are obtained from historical data bases acquired from commercial satellite images as well as ground based systems for known congestion situations occurring in the greater Baltimore corridor. In particular we acquire the vehicle type distributions and spacing from images taken from satellites and aerial photos from low-flying UAVs, of the US highways from the port of Baltimore. Simulated data for a range of traffi c impact assessments are generated from both the space data and fi xed and mobile ground-based geospatial sensor devices. In addition, data from simulated fights of targeted aircraft and mini UAVs equipped with video cameras and IR sensors platforms are also created to complete the system design.

We employ the NASA developed sensor web simulator software tool that was developed to simulate future climate observing systems, to serve as our virtual real time sensor web communication, command and control center (SW3C). The SW3C system enables interactive testing of the integration of space and ground based intermodal traffi c monitoring systems with freight fbw traffi c modeling predictions to assess the effects of adaptive pricing scenarios. SW3C employs reconfi gurable observational networks in conjunction with real time model predictions to provide management with decision making analysis. We use existing available low cost systems as the building blocks to design an end-to end sensor web congestion monitoring system. Our system design integrates the current international confi guration of commercial satellite remote sensing products, mobile/GPS devices, open source predictive models with high resolution GIS regional maps and decision analysis tools modifying freight fbw behavior based on real time congestion pricing.

We also employ the DynaMIT [1] simulation model provided by MIT for traffi c micro-simulations. Specifi c high resolution satellite imagery will be used to provide broader area traffic route coverage in identifying congestion events. Further, low cost UAVs can be directed to fly over points of congestion to provide realtime monitoring of 
events to provide data input to models to determine impact of dynamic pricing changes to accomplish the goal of dynamic congestion pricing.

Timely delivery, transit times, freight rates and delivery time reliability are considered the primary factors governing the economics of freight transportation. Other parameters like inventory storage costs, fuel cost, dwell times further affect the overall logistic cost. To simulate the effect of varying these parameters and the effect of emergent properties in the network. We focus our modeling efforts on well-known chronic congestion points in the Baltimore area. The simulations model traffi c corridors and effects of spatial and temporal traffi c patterns, rush hour traffi c, and holiday traffi c, effect of road-closures, road constructions and intermittent traffi c disruptions due to crashes or breakdown. We also consider the effect of known traffi c patterns and adaptive behavior for congestion mitigation for freight carrying vehicles based on their different loading types.

\section{Sensor Web Solution for Dynamic Congestion Pricing}

The Sensor Web Simulator (SWS) is a sophisticated simulation environment that is used to test the performance of adaptive pricing algorithms on simulated and observed traffi c fbws under actual congestion conditions. The initial design studies consist of space based remote sensing, low flying aircraft and unmanned aircraft vehicles (UAV) and ground based sensors. The data from these sensors will be merged into a coherent picture of traffi $\mathrm{c}$ in real-time. Software applications, frameworks and toolkits, plus models of various sensor web components are included in the SWS. A conceptual design of the sensor web simulators functional component interactions and feedback loops are depicted in fig. 2.

We are acquiring archives of current and historical remote sensing images from commercial satellites such as Quickbird, IKONOS, Spot, Landsat 5 and 7, Aster, EOS 1, and other nations remote sensing images over and around key roads in the Baltimore urban traffi c corridor. MiniUAVs will be fbwn over selected regions at certain times of day and night to supplement the available satellite image library. In addition to the DynaMIT traffi c simulation model [1], we plan to incorporate the NCEP weather regional forecasting (WRF) model data in the sensor web simulator along with the US Census TIGERLINE GIS database for roads and bridges. We also plan on acquiring higher resolution NAVTECH GIS data for more realistic modeling scenarios.

\begin{tabular}{|l|ll|}
\hline User Interface & $\begin{array}{l}\text { (i) } \\
\text { (ii) }\end{array}$ & $\begin{array}{l}\text { Provides an interface to configure and control a simulation experiment } \\
\text { Allows user to visualize the simulation and view analysis results }\end{array}$ \\
\hline Simulation Control & $\begin{array}{l}\text { (i) } \\
\text { (ii) }\end{array}$ & $\begin{array}{l}\text { Drives the simulation process using a discrete event engine } \\
\text { Simulation engine advances the model of the sensor web in time }\end{array}$ \\
\hline Simulation Decision Analysis & $\begin{array}{l}\text { (i) } \\
\text { (ii) } \begin{array}{l}\text { Uses detailed GIS data Models how a sensor web is structured and behaves } \\
\text { Composed of several large subsystems that perform data collection of the environment, } \\
\text { data assimilation and traffi c predictions, congestion analysis, observation targeting, and } \\
\text { communication and asset command }\end{array}\end{array}$ \\
\hline Simulated Observation Generator & (i) $\begin{array}{l}\text { Models or utilizes actual observed conditions to simulate real observation data at a specific } \\
\text { time, location and event } \\
\text { Allows an observing system to collect simulated measurements and derived data products, } \\
\text { such as image data from Satellites, UAVs, traffi c data from VeriWise and other spatial } \\
\text { ground systems } \\
\text { Makes use of DynaMITs simulation processes to generate synthetic data with realistic } \\
\text { characteristics }\end{array}$ \\
\hline
\end{tabular}

TABLE I

MAJOR Subsystems OF THE Simulator

The Sensor Web Simulator is comprised of several large subsystems. The detailed functions of the Sensor web simulator subsystems are shown in table I. A graphical front-end is provided for the confi guration, control, command, communication, and dynamic pricing. Visualizations of the simulation facilitates simplifi ed user interaction in the SWS. The user can then set up different pricing scenarios for highways and rails and execute the models to see the impact of marketing pricing on traffi c behavior. Visualization tools will be integrated to show simulation progress, providing the user with graphic displays that can show predicted traffi c states, asset locations, fight and rail paths, 


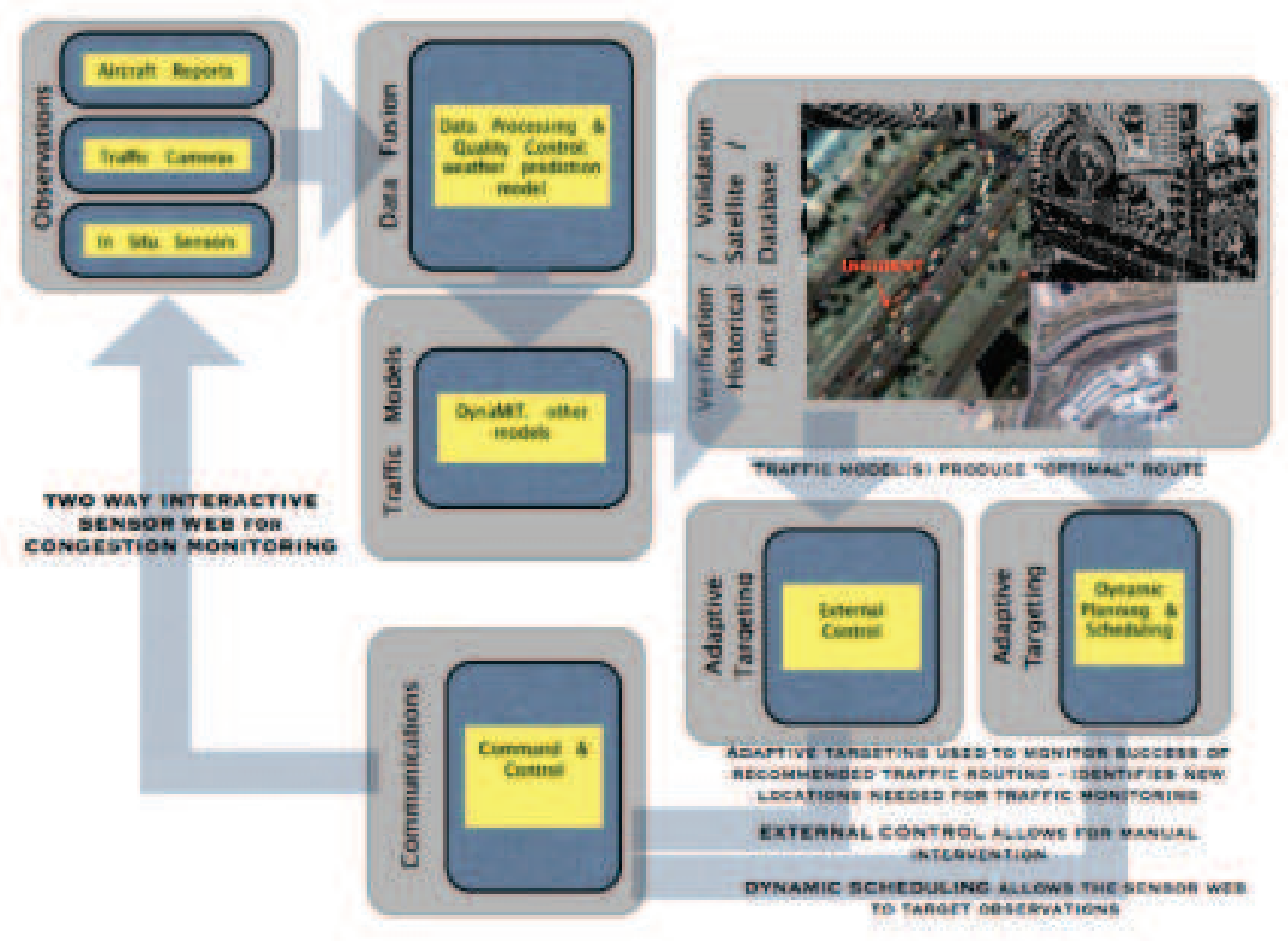

Fig. 2. Sensor Web Simulator Components and Feedback Loops

ground tracks, and communication links. The simulator will provide a set of statistical tools for comparison and analysis of those simulations results and historical database validations.

\section{Modeling Freight Traffic Flow}

We will focus on well-known chronic congestion points in the Baltimore area that we will be modeling. The simulations will model traffi c corridors and effects of spatial and temporal traffi c patterns, rush hour traffic, and holiday traffic, effect of road-closures, road constructions and intermittent traffic disruptions due to crashes or breakdown. We will also consider the effect of known traffi c patterns and adaptive behavior for congestion mitigation for freight carrying vehicles based on their different loading types.

\section{A. Space-based Information Systems}

A variety of commercial and national satellites are used to acquire high resolution remote sensing images of traffi c over the greater Baltimore corridor in the visible and other regions of the spectrum. In particular, we are identifying targets of opportunity from current and future polar low earth orbiting multi-spectral and hyper-spectral satellite sensors such as Quickbird, IKONOS, SPOT, Orbview-3 as well as from national and international satellites like NASAs EO 1, Landsat 7/8, ASTER to create our archive. While remote sensing from polar orbiting satellites cannot yield continuous monitoring of congestion because of repeat viewing orbits and cloud contamination in the visible they can provide historical patterns of traffi c congestion over wide areas resulting from unique events. We will also explore the ability to fuse the multi-spectral data of MODIS on TERRA and Aqua to assess potential changes in the concentrations of Nitrous Oxide (NOX) and other volatile organic gases to identify regions of congestion. This collection of images will form a historical warehouse of congestion situations to aid in modeling congestion scenarios. 
Recent advances have lead to the availability of small UAVs utilizing electric propulsion and miniaturized sensor systems. We will design aerial systems and mate them with an array of sensors to create a complete custom integrated system for monitoring traffic. Miniaturization of sensors is the key to utilization of small UAVs. The improved spatial resolution of UAV imagery, complements the available satellite imagery for detailed studies of specific areas.

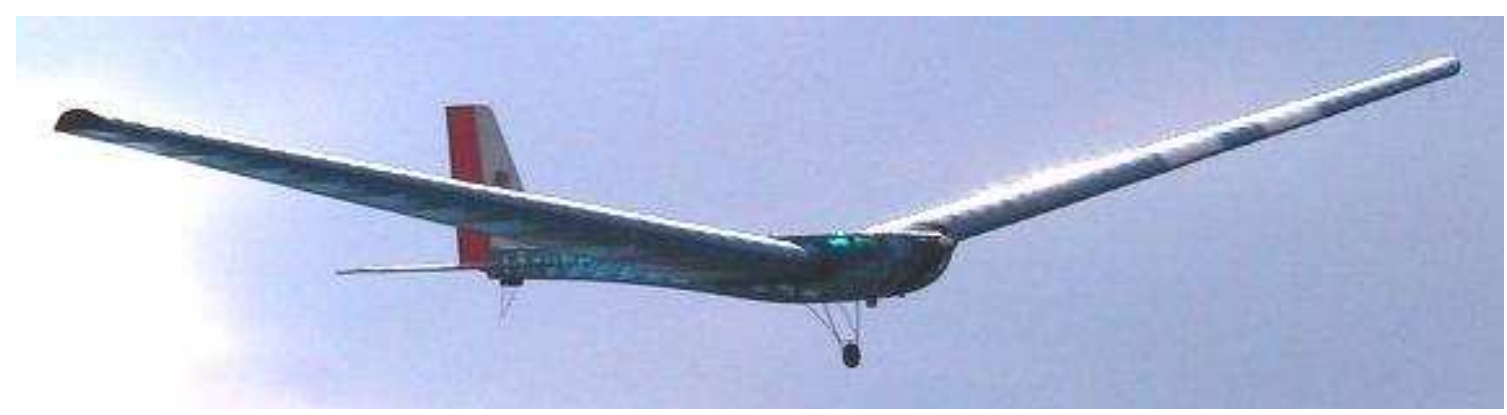

Fig. 3. A Mini-UAV

Modern batteries have dramatically improved the suitability of electric motor-based systems and results indicate that widespread use of this type of system is promising. We will acquire ultra high resolved images $(1 \mathrm{ft}$. resolution compared with 1 meter resolution) from miniUAVs equipped with video cameras, IR and LIDAR sensors that will fly over targeted roads of in the Baltimore area. Mini unmanned aerial vehicles (UAVs) can cheaply provide aerial pictures of a target road. We will purchase several of these vehicles and instrument them with video and still cameras. We will use UAVs to monitor areas of traffi c congestion where no traffi c sensor accurately measures the fbw of traffi c. These UAVs will be able monitor a large number of roads more cheaply than fi xed cameras.

\section{B. Ground-based Information Systems}

GPS and cell based mobile navigational devices like VeriWise, RFIDs, HD Video cameras, and surface monitoring devices from distributed ground platforms, provide realtime spatial-traffic information. In addition, the SW3C receives forecast data from NCEP and integrates the DynaMIT traffi c simulator employing the high resolution data. We evaluate the effectiveness of various pricing scenarios for mitigating congestion from freight fbws, particularly concentrating on the Port of Baltimore, using real and simulated data. We will develop simulations and physical prototypes of vehicle based sensor networks. These networks would use satellite feeds to provide information to the sensor network. We will build off of two models of vehicle monitoring. The first method is illustrated by the VeriWise system from GE. The second method is being developed in the StreetSmart Traffi c system. Both of theses systems use GPS satellites to identify the location of vehicles. They are differentiated in how they use and disseminate that information.

The VeriWise system from GE is a system that tracks truck trailers with a built in GPS receiver and a satellite transponder. This system is primarily used to manage assets; however the data stream from this system could be very valuable in monitoring traffi $\mathrm{c}$ through multi-modal transport. This system provides a data stream we will monitor for traffi c disruptions.

StreetSmart Traffi c is a traffi c monitoring sensor network designed to use GPS satellite feeds and ad-hoc networking. This model of communication has the signifi cant advantage that drivers do not need to pay for satellite connectivity. StreetSmart Traffi c uses system uses a combination of clustering and epidemic communication to find and disseminate traffi c information. We use a novel and new distributed clustering algorithm. Epidemic communication is modeled on the way that disease travels through a population from individual to individual. The technology behind StreetSmart Traffi c was developed at UMBC, and has been transferred to StreetSmart Traffi c Inc. We are currently developing prototype devices, with initial results expected late 2006.

We will develop both simulations and physical implementations of congestion monitoring sensor networks using space based communication infrastructure. We will study using distributed peer to peer ground based communication, centralized satellite communication and hybrid systems using both technologies. The simulations will test the effect of large scale deployment of the proposed systems. The prototypes will be used to demonstrate the feasibility of the 
proposed approach to serve as a basis for the next generation ITS implementation. These systems of sensor networks could optimize the use of existing transportation infrastructure at little cost to the Department of Transportation.

\section{ViI. Micro-Simulation Studies of Pricing Models}

Timely delivery, transit times, freight rates and delivery time reliability are considered the primary factors governing the economics of freight transportation. Other parameters like inventory storage costs, fuel cost, dwell times further affect the overall logistic cost. To simulate the effect of varying these parameters and the effect of emergent properties in the network, we will incorporate traffi c movement patterns and traffi c volumes from historical databases. We will perform traffi c micro-simulations using traffi c simulators like DynaMIT and SUMO [5] to study the effects of emergent properties leading to congestion and the effectiveness of various pricing models used to mitigate congestion. One of the important contributions of our work will be the incorporation of real-time information on a macro-level view of regional conditions provided by UAV networks and Satellite-based Sensor Web.

Since our focus is on mitigating freight congestion, which roughly represents $15 \%$ of total traffi c, we will simulate with freight carriers of various loading capacities as a percentage of total vehicular traffic. Re-routing along paths that avoid particular times of fhsh crowds for ball games and conventions (unless where necessary) can signifi cantly improve traffi c congestion and provide savings in time, fuel cost and road and vehicle wear and tear. Adaptation to current conditions will be achieved using a cost model which considers time of travel, distance, cost of tolls and congestion and weather conditions along the planned route. In the event of unforeseen hazards or other emergent traffi c conditions alternate routes can be recommended via telematic services in conjunction with GPS systems to help locate the individual freight carrier positions. We will feed the simulation with traffic data provided by Maryland Department of Transportation, the GE VeriWise system and the Maryland Port Authority. We will study the effect of dynamic congestion pricing on the behavior of freight traffic. The DynaMIT model lets us simulate the traveler behavior under various pricing congestion schemes. We plan to incorporate satellite based remote-sensing imagery into the detailed road network representation enabled by DynaMIT. DynaMIT is capable of distinguishing between vehicle types, detailed microsimulations and optimal use of historical data to generate reliable estimates of travel times.

\section{InCENTIVE-BASEd PRICING STUdies}

We focus on a cost model suitable for mitigating freight congestion by incorporating local road events, public event calendars, road segments and congestion patterns. We seek to provide a minimal cost solution to optimize and regulate the fbw of freight in a real-time and adaptive manner using real-time congestion information integrated from geo-based and satellite based sensor webs, along with GIS data and local event calendars. The pricing system will take into account the following factors: (i) time of day, (ii) current congestion, (iii) weather conditions, (iv) large public events, and (v) pollution. We consider time delay, rerouting cost, current freight locations for transit between source and destination of each freight container. Local sensors can provide immediate and local sensor information to devices that may be delayed via other means of delivery.

\section{Summary AND Future Work}

A sensor web simulator (SWS) developed and under test at NASA GSFC is being modifi ed by the UMBC team herein to conduct extensive simulation studies employing the DynaMIT traffi c model. Input to this simulator will be observations using real time simulated data acquired from actual traffi c patterns obtained from historical data bases and commercial satellite images under known congestion situations for the greater Baltimore corridor. Simulated data will also be generated from fi xed and mobile ground-based geospatial sensor devices and simulated fights of targeted aircraft and mini UAVs equipped with video cameras and IR sensors platforms to explore the range of traffi c impact assessments. We will identify a set of congestion based freight fbw pricing scenarios, both fi xed and intermittent in time and location based on natural and human induced situations. These congestion based pricing scenarios will be tested in simulation studies of potential future congestion caused by natural and human events. Preliminary results will be available for the camera ready version Dec.22, 2006 and more complete studies will be presented at the conference. 


\section{A. Acknowledgments}

The authors would like to express their appreciation to E. Lewis, Assistant Secretary MDOT and R. Burns, Director of Planning, MPA for pointing us to this critical port freight fbw problem and for their continued support for this project. We next wish to thank our colleagues at UMBC, A. Joshi, T. Seidman and P. Charalambides for their valuable comments and suggestions on integrating the varied technologies into sensor web system. We also thank J. Salvo, GE Research for offering the VeriWise devices and their available data archive and M. Jha, Earth Tech/MIT for his guidance on the implementation of the DynaMIT simulation model. We also thank G. McConaughy, NASA/GSFC for her input on adapting the Sensor Web simulator from satellite studies to traffic studies and P. Coronado, NASA/GSFC for his valuable discussion on instrumenting and fight utilization of low cost UAV's for traffi c surveillance.

\section{REFERENCES}

[1] M. Ben-Akiva, M. Bierlaire, H. N. Koutsopoulos, and R. Mishalani. Real time simulation of traffi c demand-supply interactions within dynamit.

[2] S. Dornbush. Streetsmart traffi c: Discovering and disseminating automobile congestion using vanet's. Master's thesis, University of Maryland Baltimore County, August 2006.

[3] G. Electric. Veriwise satellite asset tracking, 2006.

[4] R. L. Flanagan. Speech by Maryland Secretary of Transportation, Public Investments in Transportation: The Role of MDOT, September 2006.

[5] D. Krajzewicz, G. Hertkorn, and P. Wagner. An example of microscopic car models validation using the open source traffi c simulation sumo. 5th International Conference on ITS, 2005.

[6] T. Lomax and D. Schrank. Urban mobility study. Technical report, Texas Transportation Institute, 2005. 